\title{
Intrinsic Defects in MoS2 Grown by Pulsed Laser Deposition: From Monolayers to Bilayers
}

Bertoldo, Fabian Felix; Unocic, Raymond R; Lin, Yu-Chuan; Sang, Xiahan; Puretzky, Alexander A; Yu, Yiling; Miakota, Denys Igorevich; Rouleau, Christopher M; Schou, Jørgen; Thygesen, Kristian Sommer Total number of authors:

12

Published in:

ACS Nano

Link to article, DOI:

10.1021/acsnano.0c08835

Publication date:

2021

Document Version

Publisher's PDF, also known as Version of record

Link back to DTU Orbit

Citation (APA):

Bertoldo, F. F., Unocic, R. R., Lin, Y-C., Sang, X., Puretzky, A. A., Yu, Y., Miakota, D. I., Rouleau, C. M., Schou, J., Thygesen, K. S., Geohegan, D. B., \& Canulescu, S. (2021). Intrinsic Defects in MoS, Grown by Pulsed Laser Deposition: From Monolayers to Bilayers. ACS Nano, 15(2), 2858-2868.

https://doi.org/10.1021/acsnano.0c08835

\section{General rights}

Copyright and moral rights for the publications made accessible in the public portal are retained by the authors and/or other copyright owners and it is a condition of accessing publications that users recognise and abide by the legal requirements associated with these rights.

- Users may download and print one copy of any publication from the public portal for the purpose of private study or research.

- You may not further distribute the material or use it for any profit-making activity or commercial gain

- You may freely distribute the URL identifying the publication in the public portal 


\section{Supporting Information: Intrinsic Defects in}

\section{$\mathrm{MoS}_{2}$ Grown by Pulsed Laser Deposition: From Monolayers to Bilayers}

Fabian Bertoldo, ${ }^{\dagger}$ Raymond R. Unocic,, Yu-Chuan Lin, ${ }^{\ddagger}$ Xiahan Sang, Alexander A. Puretzky,, Yiling Yu,,${ }^{\S}$ Denys Miakota,, Christopher M. Rouleau, ${ }^{\S}$ Jørgen Schou, $\|$ Kristian S. Thygesen, ${ }^{\dagger}$ David B. Geohegan,${ }^{\S}$ and Stela Canulescu* ${ }^{*}$ $\dagger C A M D$ and Center for Nanostructured Graphene (CNG), Department of Physics, Technical University of Denmark, 2800 Kgs. Lyngby, Denmark $\ddagger$ Center for Nanophase Materials Sciences, Oak Ridge National Laboratory, Oak Ridge, Tennessee 37831, United States

IState Key Laboratory of Advanced Technology for Materials Synthesis and Processing, Wuhan University of Technology, 430070 Wuhan, China

$\S$ Center for Nanophase Materials Sciences, Oak Ridge National Laboratory, Oak Ridge, Tennessee 37831, United State

||Department of Photonics Engineering, Technical University of Denmark, 4000 Roskilde, Denmark

E-mail: stec@fotonik.dtu.dk 


\section{Supplementary Note 1: Analysis of Point Defects}
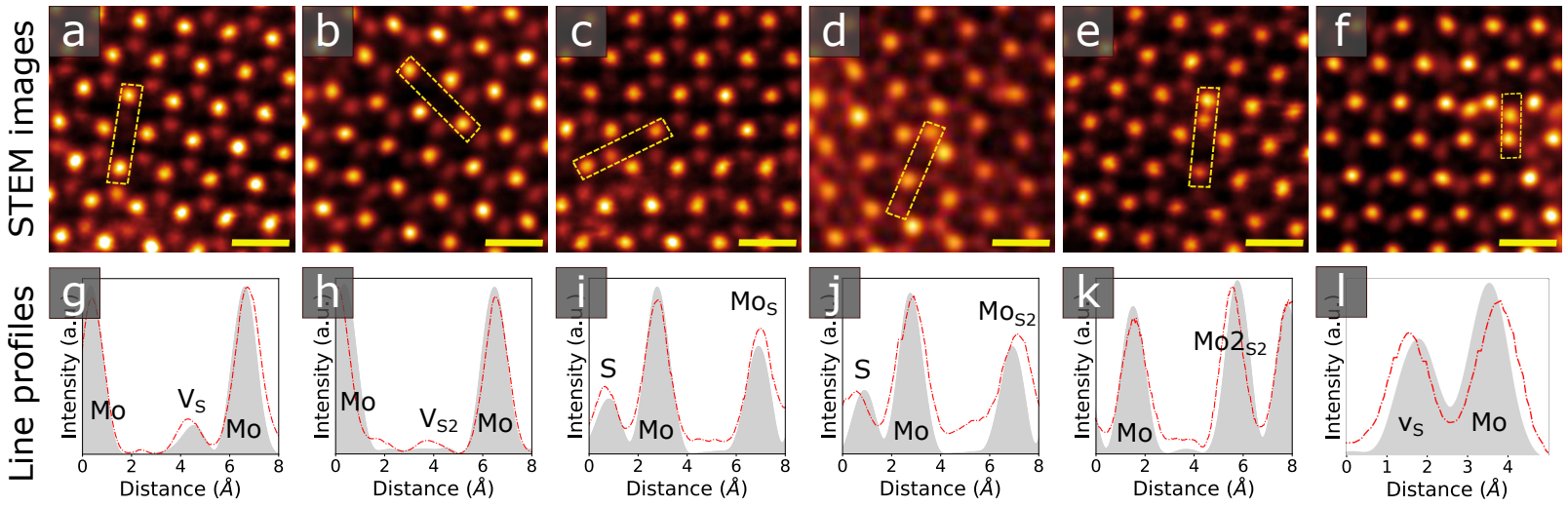

Figure S1: Experimental ADF STEM images and line profiles of point defects in $\mathbf{M o S}_{2}$. (a-f) Experimental STEM images for (a) $\mathrm{V}_{\mathrm{S}}$, (b) $\mathrm{V}_{\mathrm{S} 2}$, (c) $\mathrm{Mo}_{\mathrm{S}}$, (d) $\mathrm{Mo}_{\mathrm{S} 2}$, (e) $\mathrm{Mo}_{\mathrm{S} 2}$ and (f) $\mathrm{Mo}_{\mathrm{S}}-\mathrm{Mo}_{\mathrm{S}}$ antisite complex. (g-l) Comparison between the experimental (red dashed lines) and theoretical simulations (gray curves). The experimental line profiles were extracted from the yellow dashed boxes. The yellow scale bars have a length of $5 \AA$.

Line Profiles for Defect Identification. Figure S1 (g-l) shows the experimental and simulated line profiles of point defects in monolayer $\mathrm{MoS}_{2}$. The interatomic distances and intensity ratios between theoretically predicted and experimentally measured STEM images show a good agreement. The presence of antisites in $\mathrm{MoS}_{2}$ results in abnormal intensity variations in both simulated and experimental images. To account for the difference in the image contrast, each image was normalized to its maximum peak intensity. For example, $\mathrm{Mo}_{\mathrm{S} 2}$ appears brighter than Mo in its corresponding image, thus the line profiles were normalized to the highest intensity column. Next, the defects were identified by comparing intensity ratio between Mo and the antisite column.

Chemical Potential Limits for Formation Energies. To further strengthen the identification of point defects we calculated formation energies for the most relevant point defects identified in this work. However, when calculating formation energies of defects, it is always important to account for the energy that is needed to either add or remove atoms from the pristine structure. Thus, it is vital to look at the chemical potential limits for different 
atomic species. For our example of $\mathrm{MoS}_{2}$, in thermodynamic equilibrium we can write:

$$
\mu_{M o}+2 \mu_{S}=\mu_{M o S_{2}}
$$

where $\mu_{i}$ with $i \in\{\mathrm{Mo}, \mathrm{S}\}$ are the chemical potentials of a particular species. Let us now reference the chemical potential to the standard states:

$$
\Delta \mu_{M o}+2 \Delta \mu_{S}=\Delta H_{f}\left(M o S_{2}\right)
$$

The heat of formation of $\mathrm{MoS}_{2}$ is given by $\Delta H_{f}\left(M o S_{2}\right)=-0.92 \mathrm{eV} /$ atom which corresponds to $\Delta H_{f}\left(M o S_{2}\right)=-2.76 \mathrm{eV}$. With this knowledge, we can now look at two limiting cases:

1. S-rich limit (Mo-poor). For the S-rich limit we fix $\Delta \mu_{S}=0$ and, thus, can calculate:

$$
\begin{gathered}
\mu_{M o}=\mu_{M o}^{r e f}+\Delta H_{f}\left(M o S_{2}\right)=-8.320 \mathrm{eV}-2.76 \mathrm{eV}=-11.08 \mathrm{eV} \\
\mu_{S}=\mu_{S}^{r e f}=-4.072 \mathrm{eV}
\end{gathered}
$$

with $\mu_{M o}^{r e f}=E_{t o t}(M o)=-8.320 \mathrm{eV}$ being the reference energy for Mo.

2. S-poor (Mo-rich). Similarly, we can fix $\Delta \mu_{M o}=0$ and end up at the S-poor limit:

$$
\begin{gathered}
\mu_{M o}=\mu_{M o}^{r e f}=-8.320 \mathrm{eV} \\
\mu_{S}=\mu_{S}^{r e f}+\Delta H_{f}\left(M o S_{2}\right) / 2=-4.072 \mathrm{eV}-1.38 \mathrm{eV}=-5.452 \mathrm{eV}
\end{gathered}
$$

with $\mu_{S}^{r e f}=E_{\text {tot }}\left(S_{48}\right) / 48=-4.072 \mathrm{eV}$ being the reference energy for S.

Here, we extracted the individual reference energies from the computational 2D materials database (C2DB). ${ }^{1}$ The formation energies for the different point defects as a function of $\mathrm{S}$ chemical potential $\mu_{S}$ and Mo chemical potential $\mu_{M o}$ are shown in Figure 6. 
Quantification of point defects. Table 1 summarizes the defect count and concentration of the most commonly observed point defects in PLD specimens. The largest defect concentration is seen for single $\mathrm{S}$ vacancies $\left(\mathrm{V}_{\mathrm{S}}\right)$, followed by $\mathrm{Mos}_{\mathrm{S}}$ antisites. Note that a substantial number of $\mathrm{V}_{\mathrm{S}}$ can be induced during electron-beam imaging. Images were atoms appear blurry were therefore not used for quantification.

Table 1: Defect counts obtained from 61 images. Each image was $3 \times 3 \mathrm{~nm}^{2}$, which corresponds to $160 \mathrm{~S}$ atoms and 80 Mo atoms.

\begin{tabular}{|c||c|c|c|c|c|}
\hline & $\mathrm{V}_{\mathrm{S}}$ & $\mathrm{V}_{\mathrm{S} 2}$ & Mo & Mo2 & Mo $2_{\mathrm{S} 2}$ \\
\hline \hline Defect count & 208 & 35 & 69 & 32 & 21 \\
\hline Defect concentration & $0.378 \mathrm{~nm}^{-2}$ & $0.064 \mathrm{~nm}^{-2}$ & $0.126 \mathrm{~nm}^{-2}$ & $0.058 \mathrm{~nm}^{-2}$ & $0.038 \mathrm{~nm}^{-2}$ \\
\hline
\end{tabular}




\section{Supplementary Note 2: Adatoms}
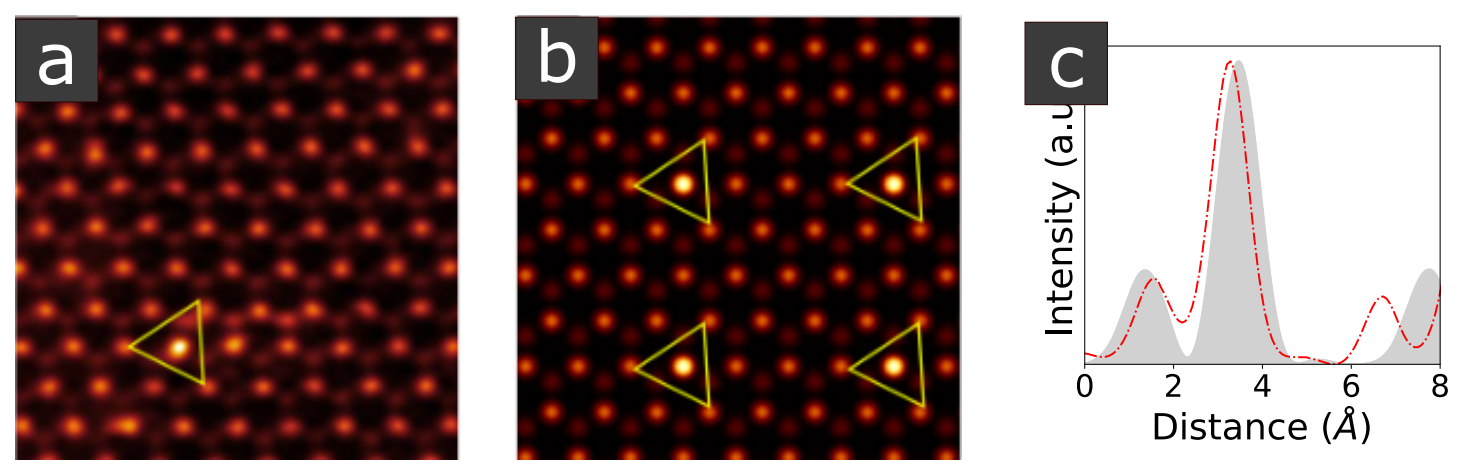

Figure S2: Mo adatoms on $\mathbf{M o S}_{2}$. Experimental (a) and simulated (b) Z-contrast STEM images of Mo adatom in $\mathrm{MoS}_{2}$ as well as their line profiles (c). Yellow triangles highlight the respective adatoms within the $\mathrm{MoS}_{2}$ structures.

Figure S2 shows the experimental and simulated ADF STEM images of Mo adatoms, as well as the comparison of the respective line profiles. We find that the Mo adatoms localize on top of the Mo atom, i.e., a-MoMo. The comparison between theory and experiment shows that Mo adatoms appears much brighter in the $Z$-contrast images, with an intensity ratio between a-Mo $\mathrm{Mo}_{\mathrm{o}}$ and Mo columns larger of 2 (Figure S2 (c)).

A large number of $\mathrm{S}$ adatoms on Mo sites $\left(\mathrm{a}-\mathrm{S}_{\mathrm{Mo}}\right)$ were observed experimentally (not shown), but they could not be reproduced in simulations. 


\section{Supplementary Note 3: Grains and Grain Boundaries}
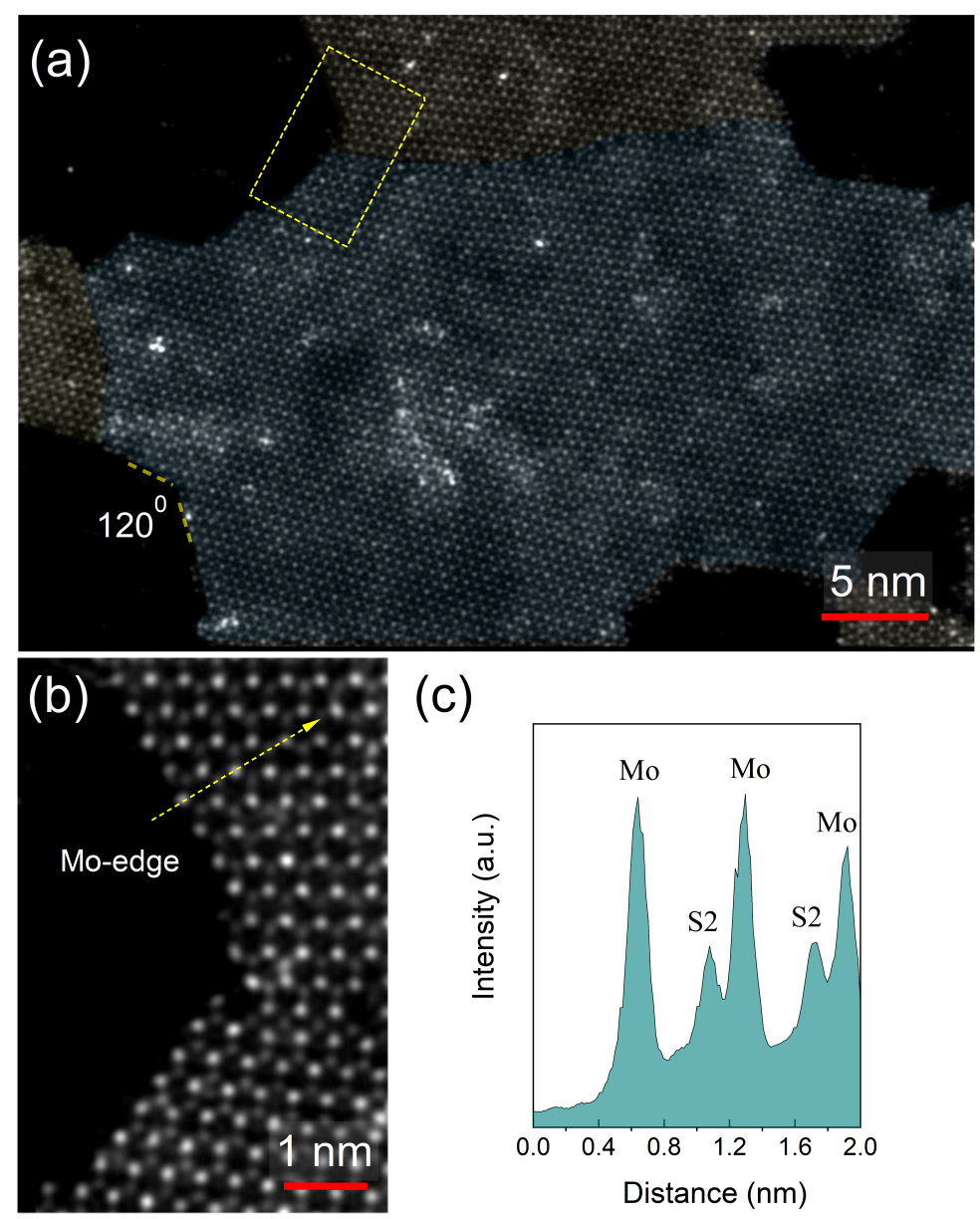

(c)

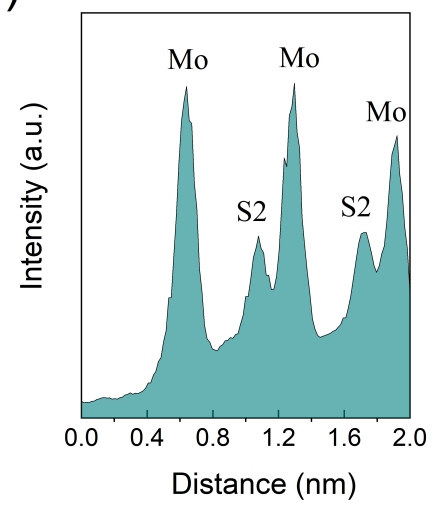

Figure S3: Grains and crystal edges in monolayer $\mathbf{M o S}_{\mathbf{2}}$. (a) Low magnification Zcontrast STEM image of monolayer $\mathrm{MoS}_{2}$ showing nanometer-size domains connected by grain boundaries. False colored regions denote individual grains. (b) Magnified view of the area highlighted in (a) showing sharp zigzag-faceted edges of the monolayer $\mathrm{MoS}_{2}$. The corresponding line profile reveals a Mo-terminated edge (c).

Figure S3 shows a polycrystalline monolayer $\mathrm{MoS}_{2}$ consisting of nano-domains connected by GBs. The edges of the monolayer are most commonly Mo-terminated, as shown in Figure S3 (c).

DFT Calculations on Grain Boundaries. In order to simulate the structural geometry of the GB with DFT we assumed periodicity to a certain extent and apply periodic boundary conditions. Therefore, we knowingly were not able to simulate the non-periodic 8-4-4-4, 8-4- 
4, 8-4 structures that have been obtained in experiment. Using periodic boundary conditions, we were able to reproduce the 8-4 periodic GB structure domains and showed that the ringlike geometry that was shown in Figure 2 is predicted to be stable in both experiment and simulation. Figure S4 shows the relaxed GB structure obtained in the supercell calculation. We can see the 71 atoms in respective supercell (black lines) as well as the alternating 8-4 ring motif. Due to the increased computational load that was necessary in order to account for the large supercell, we switched our basis in the DFT calculations from plane waves to the LCAO basis set, as well as using a $\Gamma$ point calculation. We were still using the Perdew-Burke-Ernzerhof $(\mathrm{PBE})^{2}$ functional in order to account for exchange and correlation effects.

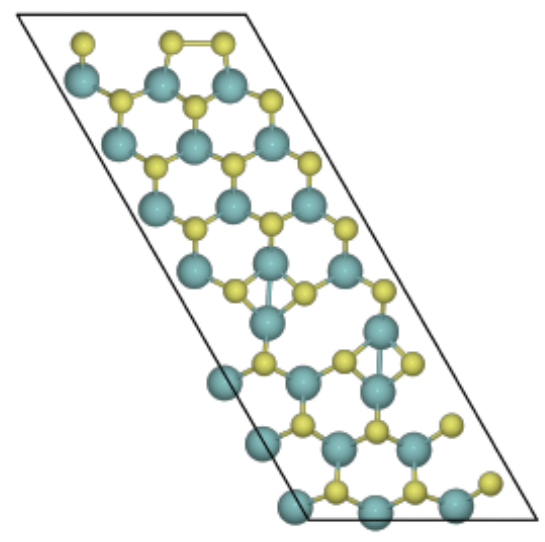

Figure S4: Unit cell for the GB simulations. Relaxed atomic structure of the $\mathrm{MoS}_{2} \mathrm{~GB}$ with 71 atoms in total. Blue: Mo atoms. Yellow: S atoms. The black rectangle highlights the unit cell that was repeated in $x$ - and $y$-directions. 


\section{Supplementary Note 4: Moiré Patterns}
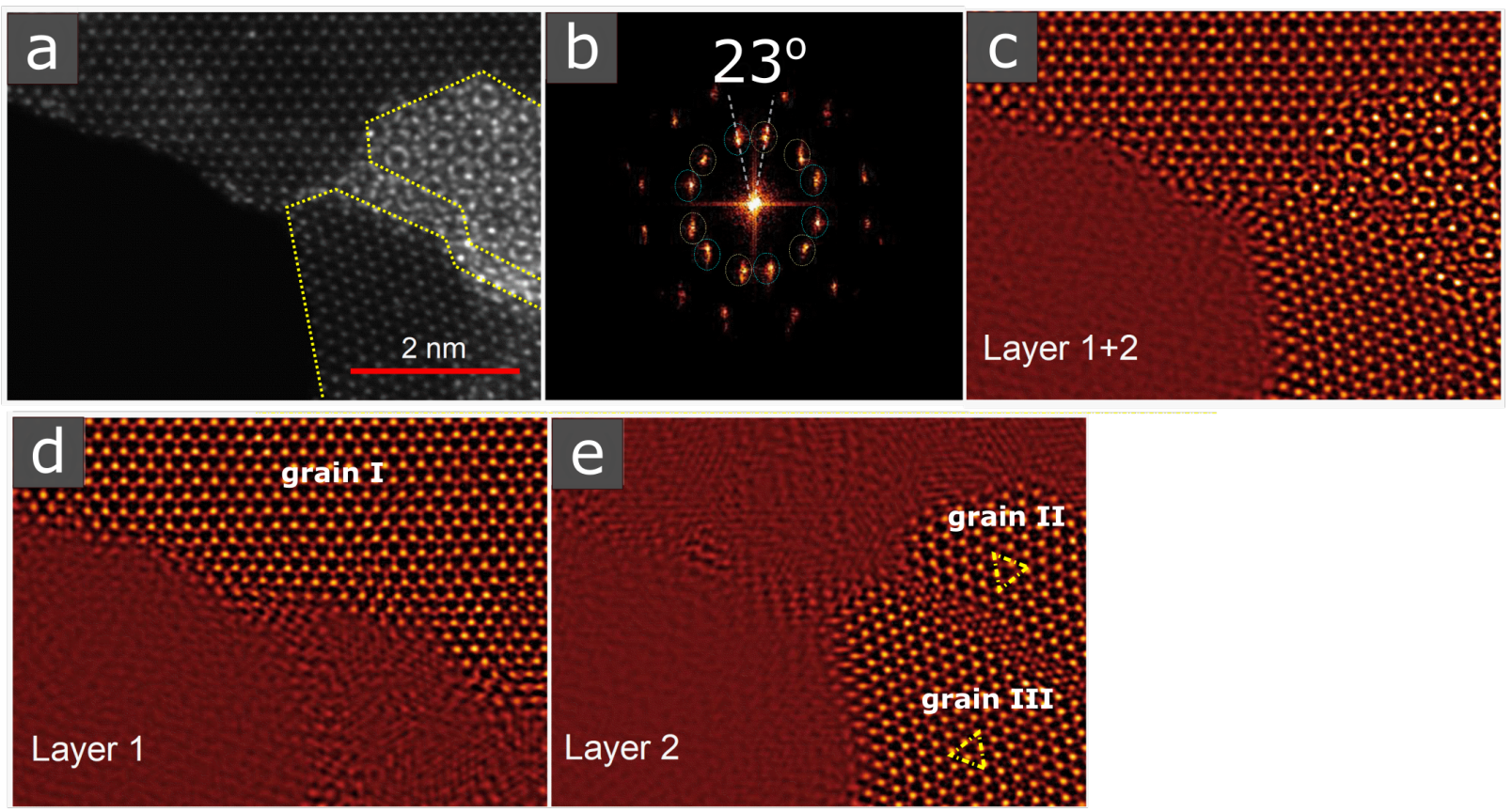

Figure S5: Atomic resolution image of a mono-bilayer $\mathrm{MoS}_{2}$ with $23^{\circ}$ twist angle in the bilayer region. (a) ADF STEM image of moiré pattern formed as a result of an overlapped junction. (b) Fourier transform (FFT) of the image showing two distinct patterns of diffraction spots with $23^{\circ}$ rotation. (d) and (e) FFT-filtered images using one set of diffraction patterns at the time showing the spatial extension of the three individual grains. (e) The sum of images (c) and (d). The yellow triangles in (e) highlight the grains II and III with $60^{\circ}$ rotation.

Figure S5 shows the $Z$-contrast STEM image of a moiré pattern with a twist angle of $23^{\circ}$. The FFT-filtered images highlight the spatial extension of the top and bottom layers in the homojunction. Here, the moiré pattern presumably emerges when grain I continues to grow on top of grain II and to a lesser degree over grain III. Figure S6 shows the ADF STEM image of a monolayer-bilayer with a $33^{\circ}$ twist angle in the bilayer region. In both cases, grain I extends over the GBs without any lattice discontinuity. 


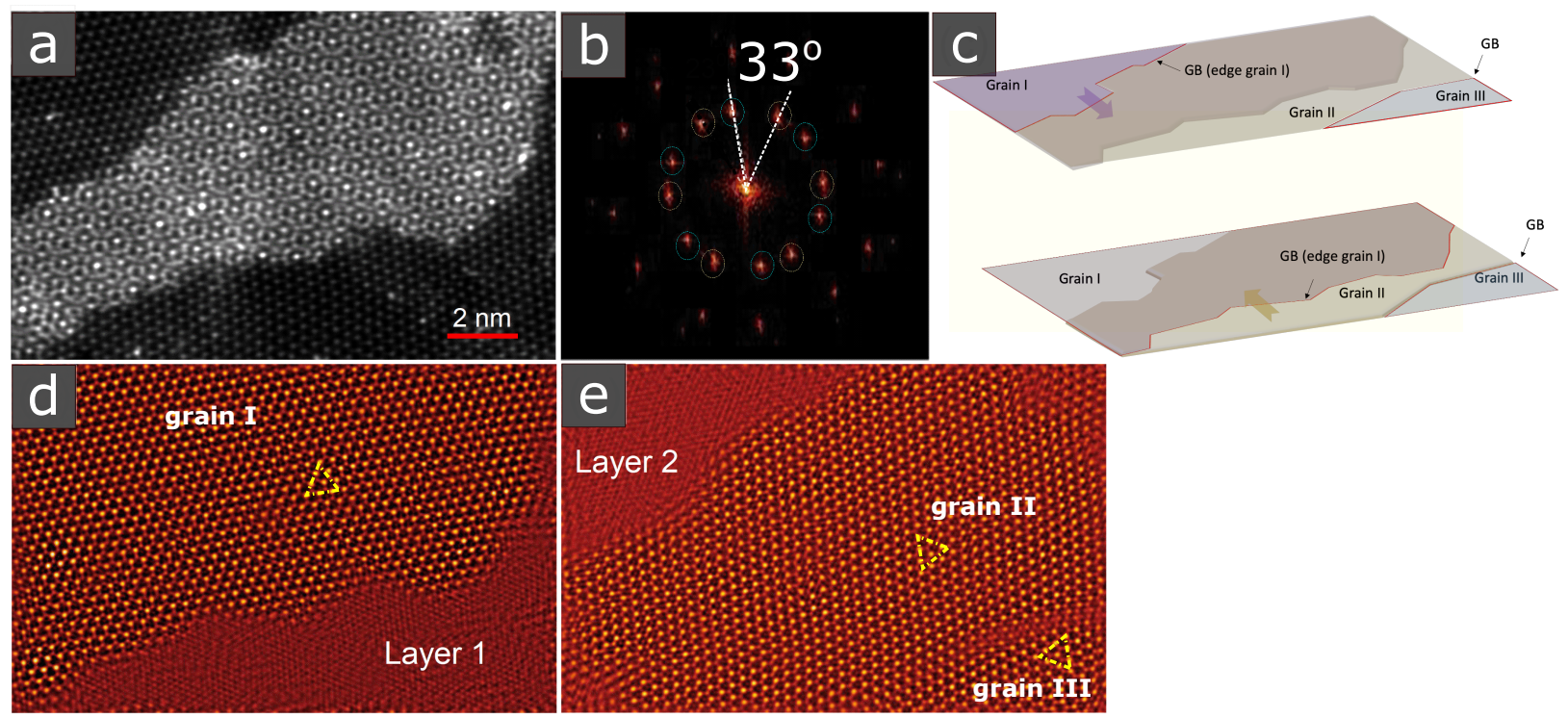

Figure S6: Atomic resolution image of a mono-bilayer $\mathbf{M o S}_{\mathbf{2}}$ with $33^{\circ}$ twist angle in the bilayer region. (a) ADF STEM image of a moiré pattern formed as a result of the overlap between grain I and II. (b) Fast Fourier transform (FFT) showing two distinct sets of diffraction spots with $33^{\circ}$ rotation. (c) Schematic views of the bilayer structure showing two possible growth mechanisms of the bilayer. (d) and (e) FFT-filtered images obtained by selective filtering each of the 6-fold diffraction spots.

\section{Supplementary Note 5: Edge Termination in AA' Stacked}

\section{Bilayer}

Figure S7 shows a AA' stacked bilayer $\mathrm{MoS}_{2}$. The line profiles of the yellow boxes labelled as (1) and (2) are shown in Figures S7 (c) and (d), respectively. Mo-terminated edges and 50 \% S-Mo terminated edges are observed. The arrows in Figure S7 (d) indicate the shrinkage of the outermost hexagonal ring in the AA' bilayer. 
(a)

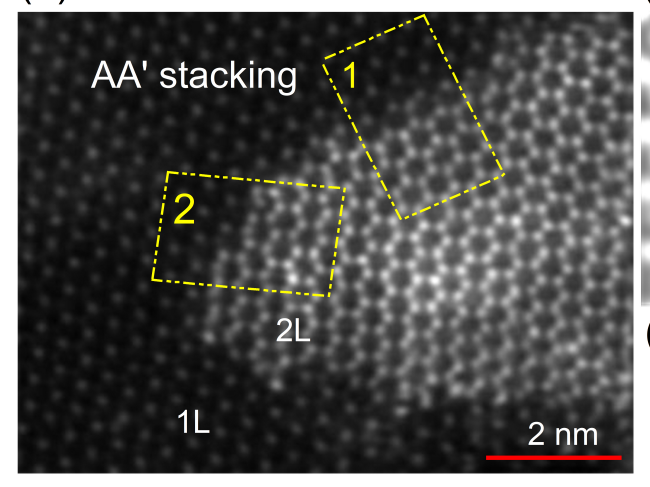

(b)

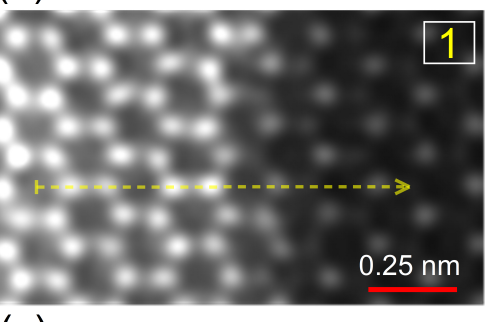

(c)

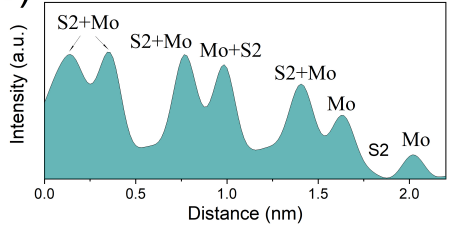

(d)

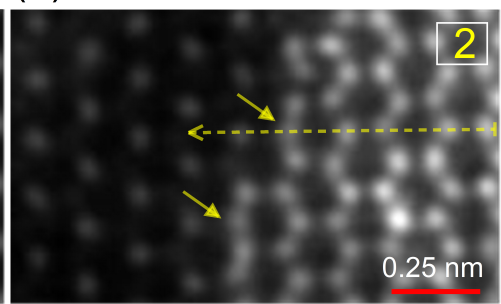

(e)

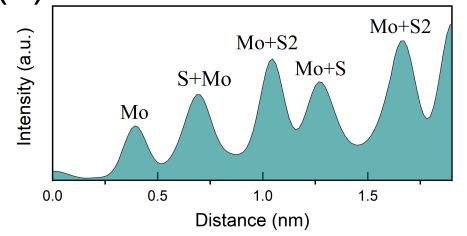

Figure S7: AA' stacked bilayer of $\mathbf{M o S}_{2}$. (a) ADF STEM image of a mono-bilayer $\mathrm{MoS}_{2}$ with AA' orientation in the bilayer region. (b) and (d) Magnified views of regions 1 and 2, respectively. The corresponding line profiles showing a Mo-terminated edge (c) and a $50 \%$ Mo-S terminated edge (e).

\section{Supplementary Note 6: Processing of the STEM-HAADF}

\section{Images}

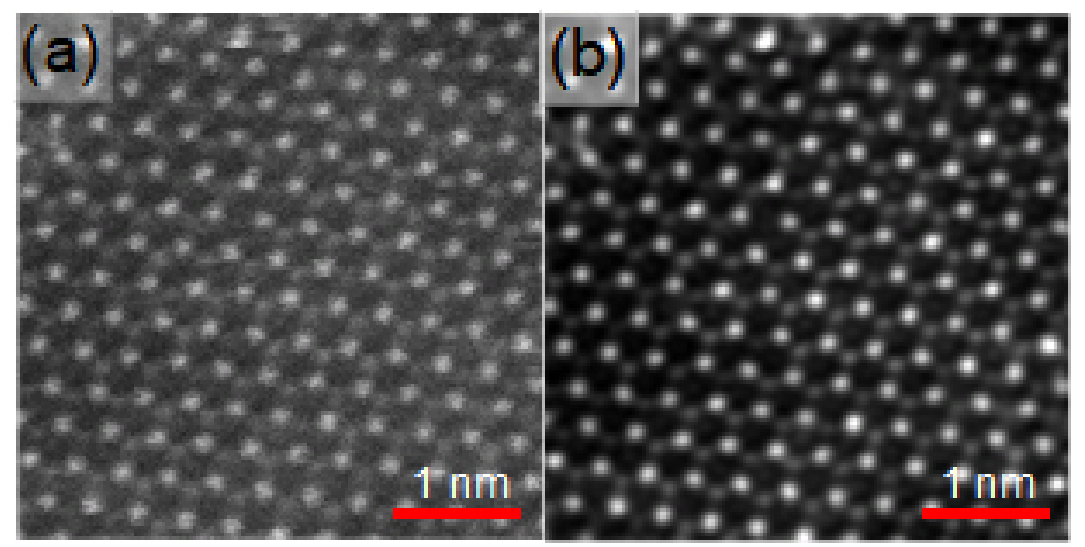

Figure S8: Processing of the ADF STEM image. Unfiltered (a) and filtered ADF STEM image (b).

The STEM images were processed using the following procedure: i) firstly, the images were smoothed using a Gaussian filtering, then ii) a fast Fourier transform (FFT) filtering was applied to the original images, and iii) the Gaussian and FFT-filtered images were multiplied. A comparison between the original and filtered ADF STEM images is shown in 
Figure S8. The noise due to electron probe tail is significantly reduced upon filtering, making the identification of S and Mo atoms or their associated point defects easily distinguishable. 


\section{Supplementary Note 7: Chemical Composition}
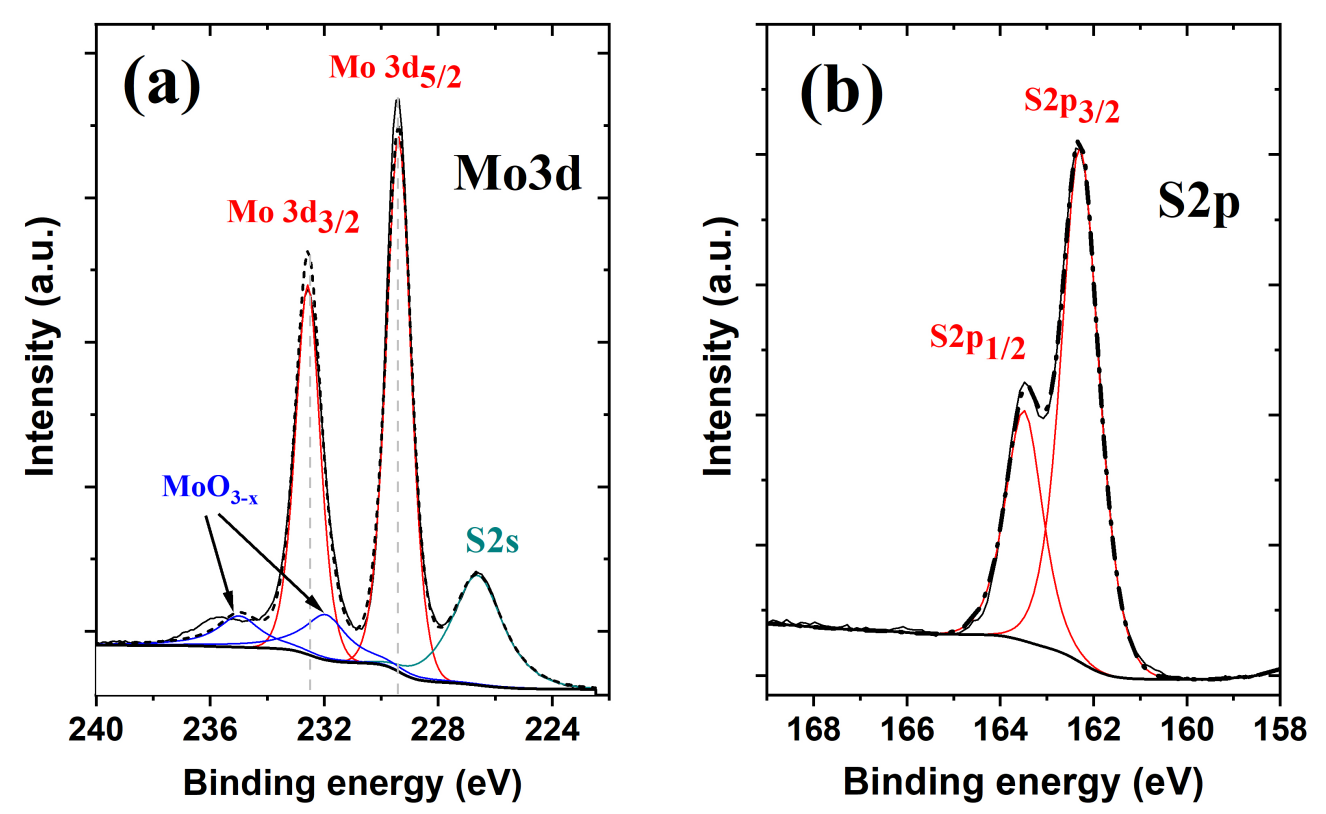

(c)

\begin{tabular}{|c|c|c|c|c|c|}
\hline & Peak & $\mathrm{BE}(\mathrm{eV})$ & FWHM (eV) & MoO3/MoS2 ratio & MoS2 stoichiometry \\
\hline 2H-MoS2 & Mo3 d5/2 & 229.39 & 1.07 & \multirow{4}{*}{0.21} & \multirow{4}{*}{2.02} \\
\hline & Mo3 d3/2 & 232.58 & 1.07 & & \\
\hline-- & S 2p1/2 & 163.49 & 0.98 & & \\
\hline-- & $S 2 p 3 / 2$ & 162.29 & 0.98 & & \\
\hline \multirow[t]{2}{*}{ MoO3-x } & Mo 3d5/2 & 231.94 & 1.8 & & \\
\hline & Mo $3 d 3 / 2$ & 234.99 & 1.8 & & \\
\hline
\end{tabular}

Figure S9: XPS spectra of monolayer $\mathrm{MoS}_{2}$ grown on $\mathrm{SiO}_{2} / \mathrm{Si}$ substrate. XPS core level spectra of Mo 3d peaks (a) and S 2p peaks (b) showing the $2 \mathrm{H}-\mathrm{MoS}_{2}$ phase and the presence of residual $\mathrm{MoO}_{3-\mathrm{x}}$ on the as-grown specimens. (c) Table showing the Mo $3 \mathrm{~d}$ and S2p peak positions, the $\mathrm{MoO}_{3} / \mathrm{MoS}_{2}$ and $\mathrm{Mo} / \mathrm{S}$ ratios

Figure S9 shows the X-ray photoelectron spectroscopy (XPS) spectra of $\mathrm{MoS}_{2}$ monolayer grown on $\mathrm{SiO}_{2} / \mathrm{Si}$ substrate. The Mo 3d doublet at higher binding energy corresponds to $\mathrm{MoO}_{3-\mathrm{x}}$. The XPS fittting yields a stoichiometric monolayer. The term BE denotes the binding energy $(\mathrm{eV})$. The film was grown at $700^{\circ} \mathrm{C}$ in vacuum. 


\section{Supplementary Note 8: Overview of Moiré Stacking}
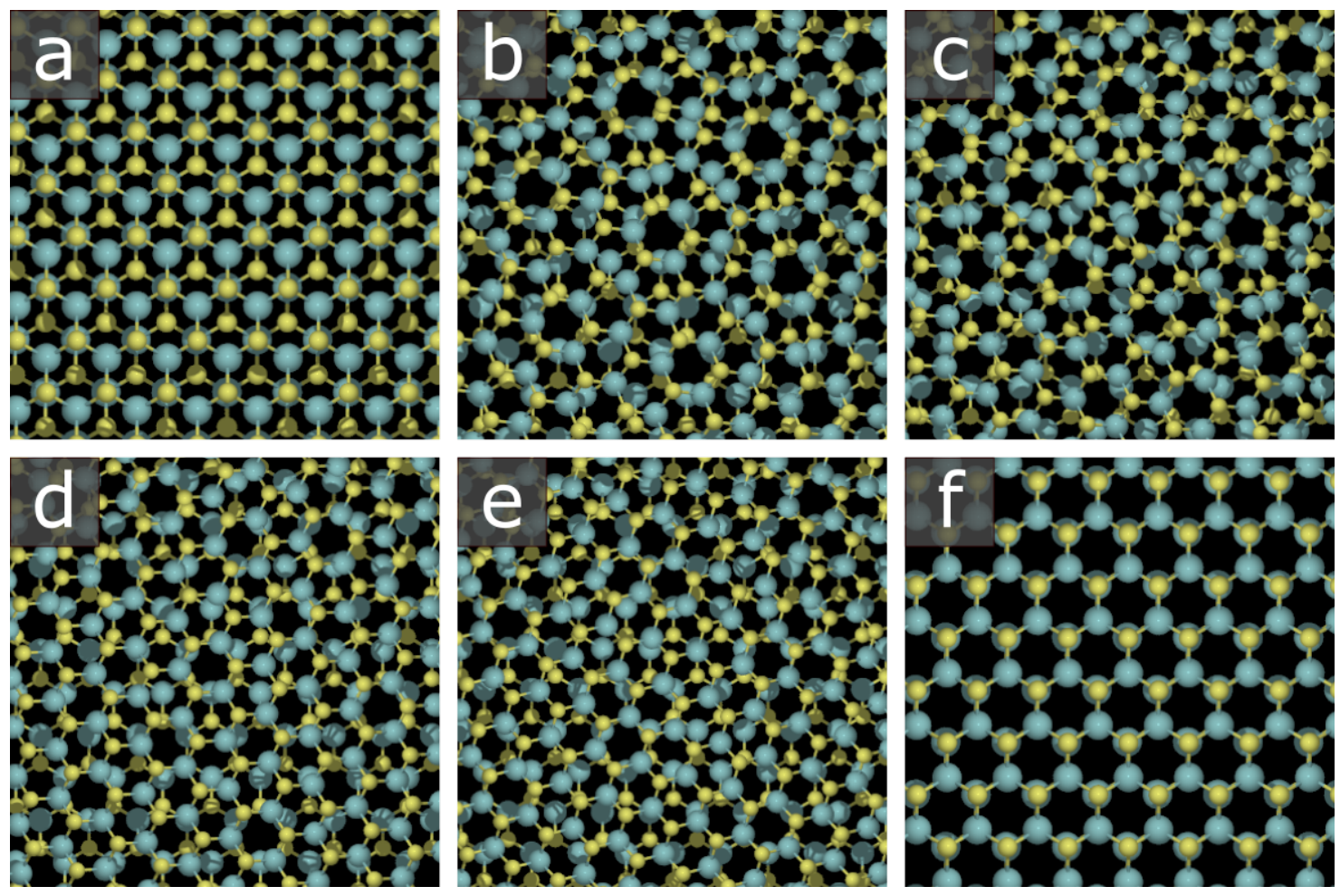

Figure S10: Atomic models of bilayer $\mathbf{M o S}_{\mathbf{2}}$. An overview of the experimentally observed

stacking in PLD-grown bilayers with the following stacking orientations: (a) $\mathrm{AB}\left(0^{\circ}\right)$, (b) $23^{\circ}$, (c) $27^{\circ}$, (d) $33^{\circ}$, (e) $44^{\circ}$ and (f) $\mathrm{AA}^{\prime}\left(60^{\circ}\right)$. 


\section{Supplementary Note 9: The $\mathrm{V}_{\mathrm{S}} \mathrm{V}_{\mathrm{S}}$ Defect Complex}

The bisulfur vacancy type complex $\left(\mathrm{V}_{\mathrm{S}} \mathrm{V}_{\mathrm{S}}\right)$ consisting of two nearby sulfur vacancies located in the upper plane of $\mathrm{MoS}_{2}$ is shown in Figure S11. The formation energy is similar to the one of $\mathrm{V}_{\mathrm{S} 2}$ (see Figure 6 ).
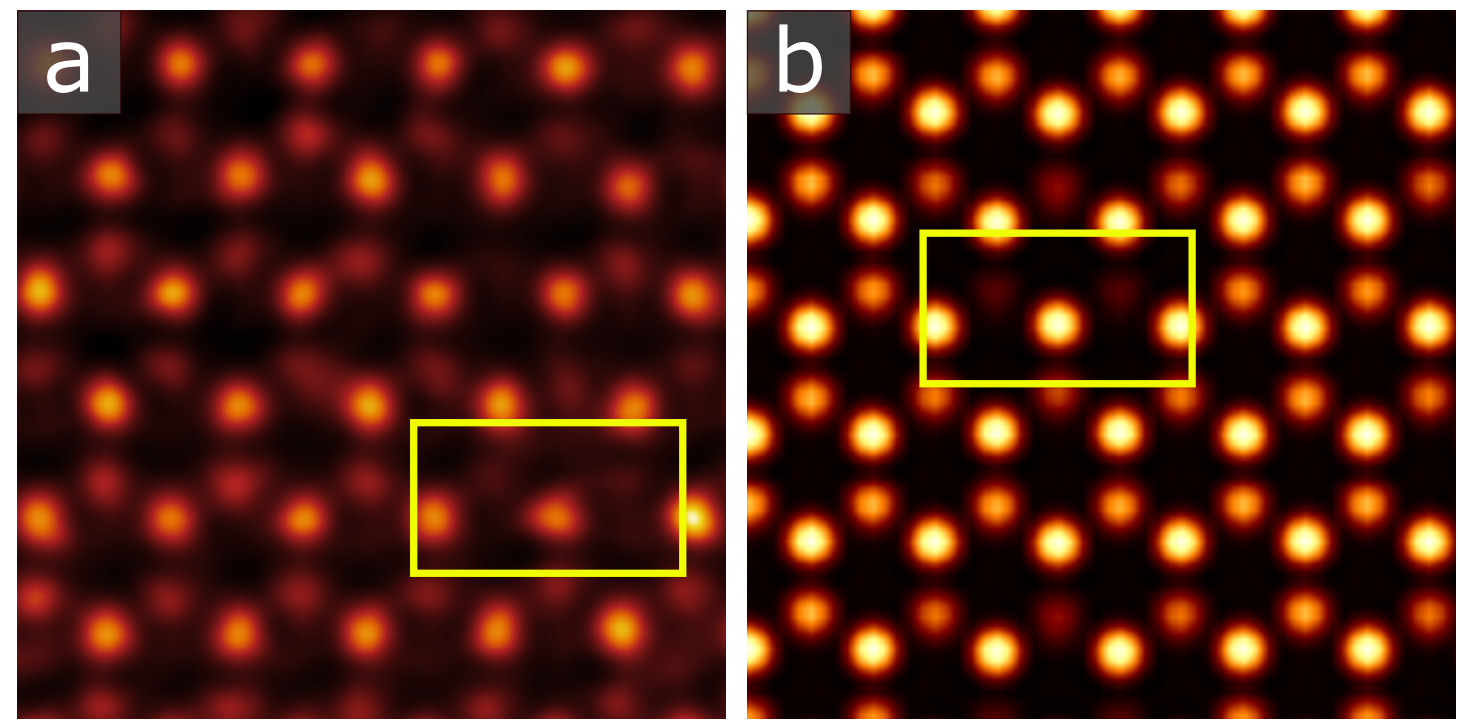

Figure S11: $\mathbf{V}_{\mathbf{S}} \mathbf{V}_{\mathbf{S}}$ defect complex. (a) Experimental and (b) theoretically simulated STEM images of the $\mathrm{V}_{\mathrm{S}} \mathrm{V}_{\mathrm{S}}$ defect complex. 


\section{References}

(1) Haastrup, S.; Strange, M.; Pandey, M.; Deilmann, T.; Schmidt, P. S.; Hinsche, N. F.; Gjerding, M. N.; Torelli, D.; Larsen, P. M.; Riis-Jensen, A. C.; Gath, J.; Jacobsen, K. W.; Mortensen, J. J.; Olsen, T.; Thygesen, K. S. The Computational 2D Materials Database: High-Throughput Modeling and Discovery of Atomically Thin Crystals. 2D Materials 2018, 5, 042002.

(2) Perdew, J. P.; Burke, K.; Ernzerhof, M. Generalized Gradient Approximation Made Simple. Phys. Rev. Lett. 1996, 77, 3865-3868. 